\title{
Visual Analytics Law Enforcement Toolkit
}

\author{
Qian Zhang, Guizhen Wang, and Jieqiong Zhao \\ Department of Science, Purdue University \\ David S. Ebert \\ Visualization and Analytics Center, Purdue University
}

\begin{abstract}
VALET, visual analytics law enforcement toolkit, is an interactive toolkit developed for law enforcement agencies to explore concerned crime information and make police resource allocation strategies. As a visual analytics toolkit, VALET is coupled with data collection, data analytics and data prediction. The objective of VALET is to assist law enforcement agencies to reduce crime rate by wisely allocating police resource based on the analytics of historical crime records. The program incorporates three steps to generate police patrol route and policeman allocation. The first step is to generate crime hotspots and crime contours of collected crime data. The next step is to analyze historical crime information and predict potential defects. Finally, the program is to compute police patrol routes and allocate police resource based on schedule and specialty. The results from the program allow us to generate risky area for different type of crimes, and evaluate policemen's performance in dealing with different type of crimes. Thus, police department is able to assign police officers to designed patrol routes that suggested by prediction tool based on policemen's specialty. This would take advantage of crime prediction and decrease the time of handling criminal activities. With VALET, law enforcement agencies are able to explore concerned crime information intelligently. At the same time, police department is prompted to allocate police resource wisely.
\end{abstract}

\section{KEYWORDS}

Crime information collection, crime prediction, police resource allocation 